\title{
Brotes de infección por Listeria monocytogenes: Una revisión de las vías que llevan a su aparición
}

\author{
M. Laura Rossi, Analía Paiva, Mariela Tornese, Sabrina Chianelli y Alcides Troncoso
}

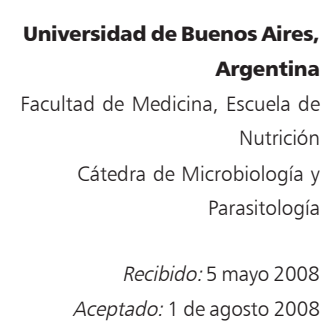

Correspondencia a: Alcides R. Troncoso microbiologiayparasitologia@yahoo.com.ar

\section{Listeria monocytogenes outbreaks: A review of the routes that favor bacterial presence}

Listeria monocytogenes is a foodborne pathogen that causes serious invasive illness, mainly in certain well-defined high-risk groups, including immunocompromised patients, pregnant women and neonates. $L$. monocytogenes primarily causes abortion, septicaemia or infections of the central nervous systems. Listeriosis outbreaks have mostly been linked to consumption of raw milk or cheese made of unpasteurized milk. Previous outbreaks of listeriosis have been linked to a variety of foods especially processed meats (such as hot dogs, deli meats, and pâté). The public health importance of listeriosis is not always recognized, particularly since listeriosis is a relatively rare disease compared with other common foodborne illnesses such as salmonellosis or botulism. However, because of its high case fatality rate, listeriosis ranks among the most frequent causes of death due to foodborne illness: second after salmonellosis. Changes in the manner food is produced, distributed and stored have created the potential for widespread outbreaks involving many countries. The pasteurization of raw milk, which destroys L. monocytogenes, does not eliminate later risk of $L$. monocytogenes contamination in dairy products. Extensive work has been ongoing in many countries during the last decade to prevent outbreaks and decrease the incidence of listeriosis. A marked reduction has occurred in its incidence in some of these countries during the 1990s, suggesting a relationship between preventive measures and reduction on human cases listeriosis.

Key words: listeriosis-dairy product- outbreak-food safety.

Palabras clave: listeriosis, productos lácteos, alimentos seguros.

\section{Introducción}

$\mathrm{L}$ a listeriosis es una infección producida por Listeria monocytogenes, un bacilo $\beta$-hemolítico, grampositivo y microaerofílico. De los siete serotipos principales, $4 \mathrm{~b}, 1 \mathrm{~b}$ y $1 \mathrm{a}$ son los responsables de la mayoría de las listeriosis humanas en E.U.A. Listeria monocytogenes está ampliamente distribuida en la naturaleza; se encuentra en heces animales, de humanos y en el suelo. A pesar de ser considerada una zoonosis que puede ser transmitida por contacto directo con animales infectados (exposición ocupacional), la mayoría de las infecciones humanas se adquieren por ingestión de alimentos contaminados y de madre a hijo intrautero o durante el parto. No tiene rasgos clínicos que la distingan de otras causas de bacteriemia.

Se pueden encontrar dos tipos de listeriosis neonatal; de comienzo precoz o tardío. En la primera, la madre puede presentar historia de enfermedad febril durante el embarazo, con parto prematuro y bajo peso al nacer. La infección en el neonato se traduce princi- palmente en septicemia, apnea neonatal o dificultad respiratoria, hepato-esplenomegalia y exantema. Usualmente los hemocultivos son positivos. La mayoría de los casos mortales tienen signos de infección sistémica, pero no todos tienen lesiones viscerales. En algunos casos, las lesiones viscerales no están diseminadas ampliamente y los estudios microscópicos revelan abscesos en vez de granulomas. Las lesiones se encuentran predominantemente en el hígado, el bazo y en las glándulas suprarrenales. Esta última localización concuerda con la naturaleza septicémica de la enfermedad intrauterina $^{1}$. En la listeriosis tardía no aparece complicación obstétrica alguna, el parto es de término y el peso del RN es normal. La enfermedad neonatal generalmente aparece entre el $5^{\circ}$ y $14^{\circ}$ día de vida y se caracteriza por problemas alimentarios, irritabilidad, fontanela anterior tensa y meningitis o meningo-encefalitis. A menudo el hemocultivo es negativo.

En su reciente revisión de datos referentes a la listeriosis humana, el Grupo de Trabajo de la Organización Mundial de la Salud, considera que la infección alimentaria se transmite predominantemente de forma 
mono-zoótica. Porque, aunque el suelo sea la fuente, la transmisión al hombre se produce principalmente desde el medioambiente a través de animales y superficies de alimentos. De este modo, L. monocytogenes debe considerarse como una bacteria medioambiental cuya transmisión al hombre se produce principalmente mediante el consumo de alimentos que se han contaminado durante su elaboración y producción ${ }^{2}$.

\section{Brotes asociados a leche}

La primera prueba seria de que los productos lácteos podían transmitir el patógeno e infectar al ser humano la proporcionaron Fleming y $\operatorname{cols}^{3}$, quienes estudiaron un brote que afectó a 49 personas, 7 casos ocurrieron en fetos o infantes y 42 en adultos inmunosuprimidos. Se implicó como fuente de infección el consumo de leche entera o parcialmente descremada, pasteurizada (odds ratio $=9,3, \mathrm{p}<0,01$ ). Aunque no se logró aislar el agente causal en los cultivos de leche, se supo que en el ganado de donde se obtuvo la leche, se habían identificado casos de listeriosis veterinaria. Todos los adultos presentaban alguna enfermedad preexistente o condiciones causantes de inmunosupresión. Los síntomas desarrollados (meningitis, septicemia o aborto) fueron variados y dependientes de la edad del paciente o del grado de inmunocompromiso y la tasa de mortalidad de los pacientes afectados fue de $29 \%$. Posteriormente se realizaron investigaciones en la planta de pasteurización, donde se vio que la leche implicada en este brote había sido procesada con el mismo equipamiento y pasteurizada en una sola planta, a tiempos y temperaturas mayores que las que indican las regulaciones de la FDA. Esta agencia gubernamental y la División de Drogas y Alimentos del Departamento de Salud de Massachusetts inspeccionaron la planta antes y después de la notificación del problema, y revelaron que las instalaciones estaban limpias, eran modernas y bien manejadas. Ningún defecto pudo haber producido una pasteurización inapropiada y no se identificaron fuentes de contaminación post pasteurización. En cuanto a la producción de la leche, ésta era provista a la planta de pasteurización por una cooperativa lechera de 450 granjas. La bacteria, que no se encontró en las muestras pasteurizadas, se aisló en 15 muestras de las 124 obtenidas antes de la pasteurización, y en dos de 14 filtros de leche. Se identificaron distintos serotipos, incluyendo aquel responsable de la epidemia. Según los veterinarios de la región, en la última primavera e inicios del verano de 1983 se diagnosticaron cuatro casos de encefalitis listeriótica bovina en vacas lecheras de granjas que suministraban leche a las cooperativas y dos en gran- jas que suministraban leche al comercio implicado.

Otro brote ocurrido a partir de leche pasteurizada es el descrito por Dalton y cols ${ }^{4}$, en el cual 45 personas que comieron en un picnic en Illinois, el 9 de julio de 1994, desarrollaron fiebre y gastroenteritis la semana posterior al evento. La enfermedad fue asociada al consumo de leche chocolatada. Se entrevistó a 82 de las 92 personas que asistieron al picnic, sobre los alimentos y bebidas consumidos en el mismo, la cantidad de leche chocolatada consumida, su temperatura y sabor. Ninguna de las personas que asistieron al picnic presentaba una enfermedad crónica o deficiencia inmune. De las 60 personas que consumieron la leche chocolatada, 45 (75\%) presentaron la enfermedad comparado con los 22 que no la tomaron y no desarrollaron síntomas. La edad media de los que presentaron la enfermedad fue 31 años (rango 3-79); y de quienes no la presentaron fue 24 años (rango 4-69). De todos, 19 (41\%) fueron mujeres y 26 (59\%) hombres. Una mujer embarazada, de 40 semanas de gestación, el día posterior al picnic tuvo un episodio de diarrea de seis horas, con ningún otro síntoma; igualmente tuvo un bebe sano. De cuatro personas que no fueron al picnic pero tomaron en sus casas leche chocolatada comprada allí, tres de ellas desarrollaron una enfermedad que coincidía con la definición del caso. Cuatro personas de 1, 7, 49 y 77 años fueron hospitalizados por un total de ocho días, pero ninguno falleció. El período de incubación medio de la infección fue 20 horas (rango: 9-32 hs) y las personas que se enfermaron tuvieron elevadas concentraciones plasmáticas de anticuerpos para listeriolisina $\mathrm{O}$. Los análisis del genoma bacteriano aislado en las deposiciones de los pacientes enfermos, de tres pacientes adicionales identificados por vigilancia, de la leche chocolatada implicada y del tanque de desagüe de la lechería, fueron indistinguibles. La listeria había sido la causa del brote.

En Europa en el período comprendido entre 1994 y el año 2000 se notificó una incidencia anual de listeriosis humana de 0,2 y 0,8 casos/100.000. En el Viejo Continente, esto se traduce en 1.600 a 8.400 casos por año, con 320 a 2.500 muertes. Este amplio rango en las incidencias puede deberse a las diferencias en los sistemas de notificación o a los brotes, que pueden aumentar marcadamente el número de casos. Entre 1991 y 2001, los casos reportados relacionados a brotes en Europa ascendieron a 2065.

En los brotes y casos esporádicos de listeriosis, las fuentes de contaminación fueron productos lácteos: desde leche cruda hasta manteca elaborada con leche pasteurizada, incluyendo varios tipos de quesos elaborados con leche sin pasteurizar (queso suave, queso fresco, queso azul de molde y queso tipo Brie $)^{5}$. 


\section{Brotes asociados a quesos}

Varios autores han descrito brotes de listeriosis provocados por el consumo de quesos. Linnan y cols ${ }^{6}$, comunicaron un brote de listeriosis relacionado con el consumo de queso estilo mexicano en el sur de California, el que afectó a 93 mujeres embarazadas o a su progenie, y a 49 pacientes no embarazadas. La evaluación de este brote indicó que la causa más frecuente de la contaminación del queso era la utilización de leche cruda en su producción. Se hace notar que los quesos blandos estilo mexicano son quesos de pasta blanda que no requieren cocción, incluyéndose dentro de esta clasificación a los conocidos con el nombre de queso blanco, queso cremoso, queso fresco, queso "de hoja", queso crema y el queso "asadero".

Boggs JD y $\operatorname{col}^{7}$, describieron también un brote de listeriosis producido entre noviembre de 2000 y enero de 2001, en Carolina del Norte, asociado al consumo de queso casero estilo mexicano, elaborado con leche sin pasteurizar, proveniente de una lechería local. Las personas afectadas eran inmigrantes mexicanos residentes en E.U.A. Sobre una entrevista inicial, la mayoría de los pacientes revelaron comer queso fresco suave estilo mexicano sin etiqueta, comprado en los mercados locales o a vendedores a domicilio. De los 12 casos identificados, 11 eran mujeres con una edad media de 21 años (18-38 años) y un hombre de 70 años inmunocomprometido. Diez de las mujeres estaban embarazadas y la infección con L. monocytogenes dio lugar a 5 fetos muertos, 3 prematuros y 2 recién nacidos infectados. La undécima mujer tenía 5 meses post parto cuando se presentó en un hospital local con meningitis causada por L. monocytogenes, sin haber tenido condición médica pre-existente alguna. Todos los casos, excepto uno, fueron confirmados por laboratorio. Fue más probable en los pacientes que en los controles, haber consumido cualquier queso comprado a vendedores ambulantes, queso fresco estilo mexicano y hot dogs. La enfermedad no fue asociada a compras y consumo en mercados o supermercados específicos de frutas o verduras crudas, productos precocidos y otros quesos como mozzarella, blue (azul o roquefort) y gorgonzola $\mathrm{u}$ otros productos lácteos. Varios miembros de la comunidad inmigrante hispánica elaboraron el queso suave fresco estilo mexicano con leche cruda, en sus hogares. Los inspectores encontraron el queso mexicano sin etiqueta en tres de los almacenes locales latinos que visitaron en Winston Salem. Los dueños de dos lecherías locales (del condado de Forsyth) divulgaron la venta de leche cruda de donde se extrajeron las muestras para su análisis y se tomaron, además, muestras de tres lecherías de condados vecinos. Se aisló el bacilo en muestras obte- nidas de nueve pacientes, en tres muestras de queso de almacenes, en una muestra de queso del hogar de un paciente y en una muestra de leche cruda de una lechería. Se condujo una investigación en una granja de vacas para determinar la fuente de la contaminación con L. monocytogenes. Se extrajeron muestras de leche de 49 vacas y del tanque de almacenaje de leche, no encontrándose positivos para L. monocytogenes. Por lo tanto, se dedujo que las vacas no estaban infectadas y que la contaminación pudo haberse originado en el medio ambiente. Como resultado de este brote, las autoridades sanitarias de Carolina del Norte prohibieron la venta de leche cruda en granjas de vacas; educaron a los comerciantes para no vender productos lácteos no regulados y recomendaron reforzar y ampliar el conocimiento de la comunidad de los peligros de comer queso fresco elaborado con leche no pasteurizada durante el embarazo. También se tomaron medidas para agregar a la listeriosis en la lista de enfermedades denunciables en Carolina del Norte.

Otro brote por alimentos contaminados con $L$. monocytogenes fue descrito por Büla y cols ${ }^{8}$. En esta oportunidad se estudiaron 57 casos ocurridos al oeste de Suiza, asociados al consumo de queso blando. De 1974 a 1982 se reportaron 28 casos de listeriosis humana. Durante el invierno de 1983 y 1984, la cantidad de casos aumentó y, llamativamente, la mayor parte de los casos se dieron en personas relativamente jóvenes y previamente sanas. Se sospechó de un origen común, pero no se lo pudo identificar. Un aumento similar ocurrió en los inviernos siguientes, hasta que en 1987 el tercer estudio de casos y controles pudo establecer una asociación con el consumo de un queso blando suizo producido en el ámbito local que se consume sólo en los meses de invierno. Dos cepas de $L$. monocytogenes fueron aisladas en los pacientes, en el queso blando suizo y en las fábricas. Desde 1983 a 1987 ocurrieron un total de 122 casos: 65 casos se dieron en recién nacidos y mujeres embarazadas; y 57 casos en varones y en mujeres no embarazadas. Todos los pacientes eran mayores de 18 años de edad, de los cuales se aisló listeria de la sangre o del LCR. Todos presentaron fiebre y $46 \%$ tuvo síntomas digestivos como vómitos y diarrea. El 83\% de los pacientes con meningitis y $59 \%$ con meningo-encefalitis experimentaron alteraciones mentales. Se observaron secuelas neurológicas en nueve (30\%) de los 27 sobrevivientes; ocho de ellos había tenido meningo-encefalitis y uno meningitis. La tasa de mortalidad fue de $32 \%$ y la edad fue la única variable significativa asociada con el riesgo de morir.

Azadian y cols ${ }^{9}$, han publicado un caso de meningitis por Listeria $\mathrm{sp}$ asociado a la ingesta de queso elaborado con leche de cabra. 
Pini y Gilbert ${ }^{10}$, examinaron 222 muestras de quesos británicos e importados y descubrieron que $10 \%$ de ellos contenía L. monocytogenes en cifras que oscilaban entre $10^{2}$ y $10^{5} \mathrm{ufc} / \mathrm{g}$.

Goulet y cols ${ }^{11}$, estudiaron los datos obtenidos del monitoreo de alimentos que realiza el Ministerio de Agricultura de Francia. Fueron muestreados 5.809 productos listos para el consumo en 1993-1994 y 6.147 en 1995-1996. Encontraron que los productos cárnicos $(19,8 \%)$ y los frutos de mar $(10,4 \%)$ estaban contaminados con mayor frecuencia que los productos lácteos $(4,7 \%)$ y las ensaladas preparadas $(4,5 \%)$. Igualmente, los productos lácteos se encontraban con mayor frecuencia contaminados con dosis más altas que otros productos. El 1,8\% de los lácteos y derivados estaban contaminados con > $100 \mathrm{ufc/gr}$, comparado con $0,3 \%$, $0,5 \%$ y $1,1 \%$ de las ensaladas, frutos de mar y productos cárnicos, respectivamente. Durante 1993-1994 y 1995-1996 observaron una disminución en la proporción de productos contaminados con $>100 \mathrm{ufc} / \mathrm{g}$.

Otro brote, descrito por Ho y cols ${ }^{12}$, implicó al queso junto con verduras crudas como responsables de listeriosis. De todos los alimentos, es evidente que los quesos son contaminados con mayor frecuencia, en especial aquellos que tienen moho blanco. Estos tienen un $\mathrm{pH}$ mayor que la mayoría de los quesos en los estadios tardíos de maduración $\mathrm{y}$, en consecuencia, son más propicios a la contaminación por esta bacteria. Hay estudios que indican que hasta $10 \%$ están contaminados con L. monocytogenes y que la prevalencia de este patógeno varía entre los diferentes tipos de quesos. En quesos blandos y semiblandos, la actividad de agua es mayor que en los duros, permitiendo el crecimiento de la bacteria. En quesos de molde, el pH se acerca a niveles neutros durante la maduración, lo que también estimula el crecimiento de L. monocytogenes. Durante la producción de lácteos fermentados, la baja actividad de agua y bajo $\mathrm{pH}$ previenen la supervivencia y crecimiento de L. monocytogenes ${ }^{5}$.

\section{Brotes asociados a alimentos no lácteos}

Además de los lácteos y sus derivados, también se encontraron otros alimentos a través de los cuales se distribuyó L. monocytogenes causando brotes.

En Canadá se dio un brote asociado al consumo de ensalada de col, afectando a 7 adultos y 34 niños recién nacidos y mujeres embarazadas, entre el $1^{\circ}$ de marzo y el $1^{\circ}$ de septiembre de $1981^{13}$. Durante el estudio se pudo acceder a una muestra de la ensalada de col que uno de los pacientes infectados conservaba en su heladera. Los resultados de los análisis mostraron la presencia de la misma cepa de L. monocytogenes en la ensalada y en la muestra de sangre extraída del paciente. El resto de los alimentos conservados en su heladera dieron negativo para la presencia de $L$. monocytogenes. El pico del brote ocurrió en los meses de verano de 1981, pero ya había habido un aumento en la cantidad de casos en el verano de 1980. Los casos que ocurrieron en mujeres embarazadas se presentaron con fiebre aguda seguida de aborto espontáneo en cinco casos, de mortinatos en cuatro casos, de nacimientos prematuros o a término pero con enfermedad grave en 23 casos o de nacimientos de niños sanos en dos casos. El índice de mortalidad en niños fue de $27 \%$. Las edades de los casos ocurridos en hombres y mujeres no embarazadas iban de los 21 hasta los 81 años. Eran seis hombres y una mujer. De los siete casos, seis presentaron meningitis y uno neumonía y sepsis. La mortalidad en los casos de meningitis fue de 33\%. El embarazo se consideró como un factor de riesgo de importancia, pero no pudo explicar el riesgo total, y en los casos de hombres y mujeres no embarazadas, con meningitis, todos eran inmunológicamente normales, lo que sugirió la importancia de otros factores de susceptibilidad no definidos. Se propuso una fuente común de L. monocytogenes, y más tarde se demostró que era posible que la fuente hubiese sido el consumo de ensalada de col o de rábanos en los tres meses previos a contraer la enfermedad. El $60 \%$ de los casos recordaban haber consumido ensalada de dicho vegetal. La misma estaba hecha por un productor de la región con repollos y zanahorias compradas a distintos mayoristas y granjeros de la zona. De la investigación se detectó como fuente responsable del brote a vegetales crudos, e identificaron que provenía de una granja que cultivaba repollo y además, tenia un rebaño de ovejas. Dos de las ovejas habían muerto por listeriosis: una en 1979 y otra en marzo de 1981. Realizaron una visita a la granja y observaron que los repollos crecían en campos fertilizados con abono y con estiércol fresco de oveja. Luego de la cosecha, que se realizaba en octubre, se procedía a una larga conservación en frío $\left(4^{\circ} \mathrm{C}\right)$, constituyendo una reserva durante el invierno y el comienzo de la primavera. Las inusuales prácticas llevadas a cabo en la granja y la reserva en frío por tiempo prolongado antes de distribuirlo a los mayoristas, permitieron que un pequeño inóculo de L. monocytogenes proliferara o causara la muerte de los microorganismos competitivos.

Un estudio señaló al paté como una posible causa de listeriosis en el Reino Unido y en la República de Irlanda $^{14}$. Entre 1967 y 1982 fueron reportados 100 casos anuales de listeriosis en Inglaterra, Gales e Irlanda del Norte. Se interrogó a los pacientes infectados a fin de obtener un registro de los alimentos consumi- 
dos tres semanas antes de contraer la enfermedad y se encontró una asociación significativa entre el consumo reciente de paté e infección con los subtipos de $L$. monocytogenes. Trece de 15 pacientes cuya enfermedad estaba causada por L. monocytogenes habían comido paté dentro de las tres semanas previas a la enfermedad. También se realizó estudios sobre los patés que se encontraban a la venta en 1989 y 1990, los cuales confirmaron la presencia de L. monocytogenes. Se advirtió que el paté de la fábrica "Y" era más propenso a estar contaminados por esta bacteria y a niveles más altos que otras fábricas. De 107 muestras de paté de la fábrica "Y", 51 (48\%) estaban contaminadas y en 12 de 50 (11\% de todas las muestras de la fábrica "Y") había un nivel de $>1.000$ ufc/g, a diferencia de las 781 muestras tomadas de otras fábricas de paté, donde 33 (4\%) estaban contaminadas y 5 de 29 (0-6\% de todas las muestras) al nivel de $>1.000 \mathrm{ufc} / \mathrm{g}$. En consecuencia, en julio de 1989 se advirtió acerca del consumo de este alimento y el gobierno suspendió la venta de paté procedente exclusivamente de la fábrica que se había identificado como origen del brote; lo que produjo un marcado descenso de la cantidad global de casos, y además se advirtió sobre los peligros para los grupos vulnerables del consumo de queso blando y sobre la necesidad de calentar las comidas cocidas enfriadas hasta que estén bien calientes antes de su consumo.

\section{Discusión}

En Europa, el número de brotes de listeriosis asociados con lácteos ya lleva aproximadamente la mitad del total de brotes. En la mayoría de los casos, estos brotes han sido ligados al consumo de leche cruda, o productos elaborados con leche cruda como quesos suaves. Aunque el primer brote científicamente probado de listeriosis transmitida por alimentos ocurrió en 1981, se sospechó que la leche cruda ha causado brotes anteriormente.

Si bien la mayoría de los casos de enfermedad se deben al consumo de leche no pasteurizada o subproductos elaborados con la misma, los brotes ocurridos a pesar de la pasteurización dejan claro que este proceso no elimina el riesgo de contaminación posterior y por lo tanto, los alimentos pasteurizados conllevan el mismo peligro que la leche cruda ${ }^{5}$. Pini y Gilbert, encontraron altos niveles de contaminación de quesos blandos con listeria. En consecuencia, consideramos inaceptables tales niveles del patógeno presente en los quesos y es evidente, como se comenta más adelante, que las pacientes embarazadas no deben incluir quesos blandos en su dieta. Se ha observado contami- nación tras la elaboración del helado, pero los datos cuantitativos son muy limitados ${ }^{10}$.

Varios estudios han probado que L. monocytogenes tolera condiciones adversas y puede, por lo tanto, sobrevivir o crecer en diferentes tipos de alimentos. Puede crecer en bajas temperaturas $\left(-1,5\right.$ a $\left.45^{\circ} \mathrm{C}\right)$ y en un amplio rango de $\mathrm{pH}(4,3$ a 9,1). También puede crecer en concentraciones salinas de hasta $10-14 \%$ y tolerar baja actividad de agua, lo que le permite sobrevivir aun en productos con alto contenido graso como quesos semiduros ${ }^{5}$. Es resistente a los medios alcalinos y tiene la capacidad de replicarse en condiciones microaerófilas y anaerobias; las concentraciones de nitrito de sodio que están permitidas en los conservantes de alimentos no inhiben la replicación del microorganismo. Sin embargo, los productos lácteos acidificados, como por ejemplo el requesón, están libres de este patógeno.

Es evidente que hay relación temporal con las medidas preventivas implementadas, de hecho la reducción de las infecciones por L. monocytogenes y la disminución de la incidencia supone una relación cau$\mathrm{sal}^{11}$.

El brote descrito por Dalton y cols ${ }^{4}$, fue probablemente causado por contaminación post pasteurización, debida a pobres prácticas sanitarias en la compañía lechera y exacerbada por mantener altas temperaturas en el camino al picnic, que permitió el rápido crecimiento de la bacteria, ya que se encontró que la leche chocolatada tenia muy altos niveles de L. monocytogenes. El vínculo entre la enfermedad invasora y la leche implicada fue confirmado en Wisconsin por electroforesis en campos pulsados. Se considera que este patógeno es más resistente a los procesos de pasteurización cuando está en el interior de un fagocito bovino que cuando está libremente suspendido en la leche. La pasteurización a alta temperatura y poco tiempo, a $71,7{ }^{\circ} \mathrm{C}$ durante 15 segundos o a $62,5{ }^{\circ} \mathrm{C}$ durante 30 minutos, puede transmitir y permitir a $L$. monocytogenes sobrevivir y replicarse ${ }^{15}$.

La pasteurización incorrecta de la leche y su contaminación posterior son las explicaciones más probables de la presencia de patógenos en la leche pasteurizada. Algunas de las bacterias de la leche producida por vacas con mastitis bovina, pueden sobrevivir a la pasteurización y replicarse a temperaturas de refrigeración. Incluso cuando se demuestra la contaminación con bacterias de la leche pasteurizada, es difícil determinar el modo y la fuente de dicha contaminación. La leche cruda es un producto intrínsecamente peligroso, y como tal, nunca debería añadirse al producto pasteurizado.

En el brote descrito por Fleming y $\operatorname{cols}^{3}$, debido a que se presume que la leche implicada en este brote 
fue adecuadamente pasteurizada, la pregunta de cuándo se contaminó asume gran importancia. Aunque la contaminación post pasteurización no puede ser excluida, parece poco difícil ya que múltiples inspecciones realizadas en la planta de producción no revelaron ninguna fuente potencial de contaminación y, además, es difícil postular un mecanismo por el cual la contaminación post pasteurización afecte sólo la leche entera y no la leche descremada, ya que ambas fueron procesadas con el mismo equipo. Por otro lado, la contaminación intrínseca de la leche y la supervivencia de algunos microorganismos, a pesar de una adecuada pasteurización, son bastante consistentes con los resultados de la investigación y biológicamente plausibles. Cuando el ganado está infectado, el organismo es excretado en la leche, y debido a que L. monocytogenes es capaz de resistir el calor, el almacenamiento post pasteurización de la leche a temperatura de refrigeración podría permitir el crecimiento selectivo de los microorganismos remanentes. De hecho, una dosis infectante relativamente pequeña podría ser la causa de que los pacientes adultos que estaban inmunocomprometidos se enfermaran. Diversos estudios han mostrado que los animales inmunosuprimidos son más susceptibles que los inmunocompetentes a un pequeño inóculo de este patógeno. Se vio que la habilidad de L. monocytogenes de existir como un parásito intracelular podría incrementar la probabilidad de supervivencia de algunos microorganismos luego de la pasteurización de la leche.

La asociación de listeriosis con el consumo de leche apoya la hipótesis de que L. monocytogenes es un patógeno transmitido a los humanos desde animales infectados o sus productos. Los resultados sugieren que la leche debería ser considerada un posible vehículo de infección en listeriosis esporádica, y que aunque la pasteurización es un método altamente efectivo para eliminar patógenos de la leche, puede no ser siempre $100 \%$ efectivo $^{3}$.

Hay datos contradictorios y poco concluyentes de los caprichos de la infección expresada en diferentes grupos de riesgo. Generalmente la expresión clínica de los casos esporádicos no parece ser diferente de la observada durante los brotes, lo que apoya el concepto de que muchos casos esporádicos son consecuencia de una transmisión alimentaria.

Sólo unos pocos sujetos desarrollan realmente listeriosis, y en los sujetos sanos, la bacteria ingerida queda, aparentemente, contenida por los macrófagos activados. No obstante, en ocasiones se produce una enfermedad clínica. La gama de enfermedades infecciosas causadas por Listeria sp se extiende desde las entidades asintomáticas hasta las potencialmente mortales, tales como la septicemia y la meningitis ${ }^{9}$.
El embarazo se considera como un factor de riesgo de importancia, pero no puede explicar el riesgo total, y en los casos de hombres y mujeres no embarazadas, con meningitis, todos eran inmunológicamente normales, lo que sugiere la importancia de otros factores de susceptibilidad no definidos.

Los hallazgos de McLauchlin proveyeron evidencia sobre una relación entre algunos casos y el consumo de paté. No se obtuvieron evidencias microbiológicas directas para asegurar una relación entre casos y consumo de estos productos alimenticios, ya que no se tenía disponibles muestras del paté consumido por los pacientes ${ }^{14}$.

En el brote descrito por Boggs ${ }^{7}$, se vio que por más que las leyes prohiban la venta y el consumo de leche cruda y de productos lácteos crudos, como en el caso de Carolina del Norte, tales prácticas persistirán en algunas comunidades, como resultado de las preferencias del gusto por este tipo de quesos, y por razones culturales. Lamentablemente, el renombre del queso fresco casero estilo mexicano elaborado con leche no pasteurizada ha dado lugar a varios brotes en comunidades hispánicas desde los años 80 , con abortos sépticos como consecuencia, atribuidos a L. monocytogenes. Además, el hecho que otros 28 estados sí permiten la venta de leche cruda a los consumidores, dificulta la educación alimentaria a la población, y como el queso en estas comunidades se produce en hogares privados, las regulaciones en el ámbito sanitario son difíciles de hacer cumplir. A esto se suma también la dificultad de comunicar exitosamente los mensajes de salud pública a la comunidad hispánica sobre el riesgo que implica el consumo de este tipo de productos debido a la lengua y otras barreras culturales y sociales.

Evidentemente, queda mucho por dilucidar en lo que respecta a los aspectos epidemiológicos de la listeriosis, ya que es un problema sanitario en evolución. Es esencial un esfuerzo conjunto por parte del nutricionista, el microbiólogo, el médico clínico y el epidemiólogo. También debe intervenir el ginecólogo, ya que con muchísima frecuencia las víctimas iniciales son la paciente embarazada y su feto intrauterino.

Una inferencia extraíble de los principales brotes epidémicos publicados es que si las epidemias de listeriosis están causadas por transmisión alimentaria, también podrán producirse por la misma vía, incluso en mayor grado, los casos esporádicos. Esta suposición concuerda con informaciones de casos aislados que vinculan la listeriosis con el aislado del tipo idéntico de fago del microorganismo en un alimento ingerido.

Aunque se desconoce la fuente de la mayoría de las infecciones por listeria, ahora es bastante convincente la prueba de la transmisión alimentaria en el hombre; 
ésta puede ser responsable de epidemias, de brotes y de casos esporádicos. Es importante mantener la perspectiva ante el problema de la listeriosis cuando se habla a las pacientes, señalando que la incidencia de listeriosis perinatal es baja y que sólo unas pocas pacientes embarazadas adquieren realmente listeriosis. Este último hecho no es un consuelo para las pacientes que continúan el embarazo y dan a luz un niño discapacitado o un mortinato. En la visita prenatal inicial hay que aconsejar a las mujeres embarazadas que no consuman leche cruda, quesos blandos y curados y que laven bien las frutas y hortalizas. Por otra parte, las comidas pre-cocinadas y las aves listas para comer no se deben consumir frías. Si se incluyen estos alimentos en la dieta, sólo deben ingerirse si se han calentado a más de $75^{\circ} \mathrm{C}$.

El Grupo de Trabajo de la Organización Mundial de la Salud, ${ }^{2}$ llegó a la conclusión, a partir de la información disponible, de que "la pasteurización es un proceso seguro que reduce el número de L. monocytogenes presente en la leche cruda a niveles que no suponen un riesgo apreciable para la salud humana". Esta declaración sólo causa preocupación a las pacientes embarazadas -el patógeno tiene predilección por la unidad fetoplacentaria- y a los pacientes inmunocomprometidos, ya que en ellos, estos bajos niveles de bacterias pueden ser perjudiciales.

Los médicos y el personal de salud pública deberían pedir que las deposiciones se analicen para detectar la presencia de L. monocytogenes en brotes de enfermedad caracterizada por diarrea, fiebre y cuadro "gripesimil". Para prevenir la enfermedad, las lecherías deberían monitorear sus productos finales, y controlar el nivel general de sanidad de la planta mediante adecuada limpieza, sanitización y mantenimiento de los equipos, según programas de análisis de peligros y puntos críticos de control. Las fechas de vencimiento deben basarse en los resultados de tales pruebas ${ }^{4}$. Sin embargo, hay que hablar a la paciente de la capacidad del patógeno para replicarse en alimentos refrigerados $\mathrm{y}$, posiblemente, en productos tratados por el calor.

Varios estudios han encontrado relación entre la aplicación de medidas preventivas en la industria alimenticia y la disminución de la incidencia de listeriosis. Datos obtenidos del monitoreo de alimentos sugieren de manera consistente que la aplicación de estas medidas reduce exitosamente la distribución de la L. monocytogenes en los alimentos listos para consumir ${ }^{14}$.

Tappero y cols ${ }^{16}$, analizaron si la reducción de la incidencia de listeriosis en E.U.A. de 1989 a 1993, se podía deber a la efectividad de las medidas de prevención que se fueron aplicando a lo largo de este período a escala industrial, de regulación y educacional. En cuanto a las medidas preventivas y su evolución a lo largo del tiempo, observaron que, luego de un brote ocurrido en Los Ángeles en 1985, la FDA comenzó a monitorear la presencia de L. monocytogenes en lácteos y derivados y se expandió un programa de control a la industria cárnica, sobre productos cocidos y listos para el consumo.

Debido a que la mayoría de los casos de listeriosis humana en E.U.A. ocurría de forma esporádica y no en brotes, el CDC, junto a la FDA, comenzó a vigilar activamente los casos esporádicos a partir de 1986 con el objetivo de detectar la causa y los posibles factores de riesgo. En 1988, el primer estudio de casos y controles, detectó como factor de riesgo el consumo de productos avícolas mal cocidos y hot dogs no recalentados, pero no consiguieron evidencia microbiológica. En 1989, detectaron un caso de listeriosis por consumo de salchichas de carne de pavo y, esta vez, sí obtuvieron evidencia microbiológica en paquetes cerrados de salchichas de pavo. En respuesta a esto, el Departamento de Agricultura de los E.U.A. instauró un programa de vigilancia y de cero tolerancia para la L. monocytogenes en productos cárnicos listos para consumo y, más tarde, se extendió a sandwiches, ensaladas listas y productos de pesca.

Por otra parte, se observó que los índices de listeriosis disminuyeron en todas las áreas vigiladas. Estimaron 1965 casos y ocurrieron 481 muertes en 1989 comparados con los 1.092 casos estimados y 248 muertes en 1993, una reducción de 44 y $48 \%$ de la enfermedad y muertes, respectivamente. Entre los adultos mayores de 50 años, el índice disminuyó de 16,2 por millón en 1989 a 10,2 por millón en 1993. La enfermedad en niños recién nacidos y mujeres embarazadas disminuyó de 17,4 casos por cada 10.000 nacimientos en 1989 a 8,6 casos por cada 10.000 nacimientos en 1993 .

La relación temporal entre los esfuerzos realizados para prevenir la listeriosis por parte de las industrias alimentarias de los E.U.A. y la regulación del gobierno y la disminución de los índices de la enfermedad, sugieren que estas medidas son responsables de esta disminución ${ }^{16}$.

Hay que informar a la población, y en especial a las mujeres embarazadas, que los alimentos cocidos deben guardarse en el refrigerador en un compartimento aparte, separados de los quesos y los alimentos crudos. Insistir en que estos alimentos se deben guardar el menor tiempo posible y que hay que respetar la fecha de caducidad de las etiquetas. Cuando se utilice un horno de microondas, los pacientes deben prestar atención a las instrucciones del fabricante para asegurar una temperatura uniforme en los alimentos. Asimismo, deben descartarse los alimentos sobrantes recalentados. Es importante que el paciente conozca estos hechos, aunque queda por determinar la medida en la 
que los casos de listeriosis son esporádicos o forman parte de un brote y la frecuencia con la que se relacionan con el alimento ${ }^{17}$.

Desdichadamente, a causa de la falta de información sobre la tasa de contaminación de los alimentos y sobre el riesgo de enfermedad invasora de las pacientes gestantes expuestas al patógeno, es difícil recomendar la eliminación de la dieta de una lista de alimentos. Por ello resulta de fundamental importancia el consejo educativo exhaustivo de los individuos que pertenecen a los grupos de riesgo que mayor probabilidades tienen de desarrollar está enfermedad y el entrenamiento de los agentes de salud para la identificación temprana de los casos sospechosos.

\section{Resumen}

Listeria monocytogenes es un patógeno que cuando se encuentra en los alimentos, puede causar serias enfermedades, principalmente en grupos de alto riesgo como inmunocomprometidos, mujeres embaraza- das y neonatos. Listeria monocytogenes es una importante causa de abortos, septicemia o infección del SNC. Antiguamente, los brotes se vinculaban con una gran variedad de alimentos, especialmente carnes procesadas (salchichas, paté, productos precocidos); en la actualidad, la mayoría se vinculan al consumo de leche cruda o quesos elaborados con leche sin pasteurizar. La importancia de la listeriosis para la salud pública no siempre es reconocida, sobre todo porque es una enfermedad relativamente rara, en comparación con otras más comunes como la salmonelosis. Sin embargo, debido a su alta tasa de letalidad, la listeriosis se encuentra entre las causas más frecuentes de muerte por enfermedades transmitidas por alimentos ocupando la segunda posición, después de la salmonelosis. Los cambios en la forma de producción, almacenamiento y distribución de los alimentos han propiciado diversos brotes. En las últimas décadas, muchos países han encaminado intensos trabajos hacia la prevención de la listeriosis; la notable reducción de su incidencia en la década del '90, sugiere una relación positiva con las medidas implementadas.

\section{Referencias}

1.- Rappaport F, Rabonowitz M, Taoff R, Krochnik N. Genital listeriosis as a cause of repeated abortion. Lancet 1960; 1: 1273-5.

2.- World Health Organization Working Group: Food-borne listeriosis. Bull World Health Org 1988; 66: 421-8.

3.- Fleming D W, Cochi S L, MacDonald K L, Brondum, Hayes P S, Plikaytis, et al. Pasteurized milk as a vehicle of infection in an outbreak of listeriosis. N Engl J Med 1985; 312: 404-7.

4.- Dalton C B, Austin C C, Sobel J, Hayes P S, Bibb W F, Graves L M, et al. An outbreak of gastroenteritis and fever due to Listeria monocytogenes in milk. N Engl J Med 1997; 336: 100-5.

5.- Lundén J, Tolvanen R, Korkeala H. Human listeriosis outbreaks linked to dairy products in Europe. J Dairy Sci 2004; 87: E6-E12.

6.- Linnan M J, Mascola L, Lou X D, Goulet V, May S, Salminen C, et al. Epidemic listeriosis associated with Mexican-style cheese. N Engl J Med 1988; 319: 823-8.

7.- Boggs J D, Whitwam R E, Hale L M,
Briscoe R P, Kahn S E. Outbreak of listeriosis associated with homemade Mexican -style cheese- North Carolina, October 2000-January 2001. MMWR Morbid Mortal Wkly Rep 2001; 50: 560-2.

8.- Büla C J, Bille J, Glauser M P. An epidemic of food-borne listeriosis in western Switzerland: description of 57 cases involving adults. Clin Infect Dis 1995; 20: 66-72.

9.- Azadian B S, Finnerty G T, Pearson A D. Cheese-borne listeria meningitis in immunocompetent patient. Lancet 1989; 1 (8633): 322-3.

10.- Pini P N, Gilbert R J. The occurrence in de UK of Listeria species in raw chicken and soft food. Int J Food Microbiol 1988; 6: 317-26.

11.- Goulet V, deValk. Effect of prevention measures on incidence of human listeriosis, France, 1987-1997. Emerg Infect Dis 2001; 7: 983-9.

12.- Ho J L, Shands K N, Friedland G, Eckind P, Fraser D W. An outbreak of type $4 \mathrm{~b} L$. monocytogenes infection involving patients from eight Boston hospitals. Arch Intern Med 1986; 146: 520-4.

13.- Schlech WF, Lavigne PM, Bortolussi RA, Allen AC, Haldane EV, Wort AJ. Epidemic listeriosis-evidence for transmission by food. N Engl J Med 1983; 308: 203-6.

14. - McLauchlin J, Hall S M, Velani S K, Gilbert R J. Human listeriosis and paté: a possible association. Br Med J 1991; 28; 303 (6805): 773-5.

15.- Doyle M P, Glass K A, Beery J T, García G A, Pollard D J, Schultz R D. Survival of Listeria monocytogenes in milk during high-temperature, short-time pasteurization. Appl Environ Microbiol. 1987; 53: 1433-8.

16.- Tappero J W, Schuchat A, Deaver K A, Mascola L, Wenger J D. Reduction in the incidence of human listeriosis in the United States. Effectiveness of prevention efforts? The Listeriosis Study Group. JAMA 1995; 273: 1118-22.

17.- Slutsker L, Evans M C, Schuchat A. Listeriosis. In: Scheld W M, Craig W A, Hughes J M, eds. Emerging Infections 4. Washington, DC: ASM Press, 2000. 